$\Rightarrow$ SMALL RNAS

\title{
A tiny stabilizer of development
}

A new paper provides the first experimental evidence that microRNAs (miRNAs) confer robustness, by showing that a Drosophila melanogaster miRNA buffers a developmental process against environmental fluctuation.

$\mathrm{Li}$ and colleagues investigated the role of miR-7 in sensory organ development. Previous studies had shown that, under uniform conditions, loss of miR-7 function has little impact on either the expression levels of its regulatory targets or on developmental outcome. Does miR-7 have a role in stabilizing sensory organ development in less stable environments?

Using transgenes, reporter assays and mutant analyses, the authors carefully dissected the interactions between miR-7, its regulators and its targets during the differentiation of photoreceptors and proprioreceptors. In both cases, miR-7 functions as part of one or more feedforward or feedback loops in a way that is predicted to stabilize the expression of key determinants of cell fate. Indeed, mir-7 mutant larvae that were exposed to temperature fluctuations showed altered expression of these genes and defects in the specification and patterning of sensory organs.

Interestingly, although miR-7 is highly conserved from flies to humans, its functions are not; for example, it is not involved in vertebrate sensory organ development. The authors suggest that conserved miRNAs might be recruited into new regulatory interactions during evolution specifically to provide robustness to regulatory networks.

Louisa Flintoft

ORIGINAL RESEARCH PAPER Li, X. et al.

A microRNA imparts robustness against

environmental fluctuation during development.

Cell 137, 273-282 (2009).

FURTHER READING Flynt, A. S. \& Lai, E. C.

Biological principles of microRNA-mediated

regulation: shared themes amid diversity. Nature

Rev. Genet. 9, 831-842 (2008) 